\title{
Formation and dynamics of quantum hydrodynamical breathing-ring solitons
}

\author{
Samuel N. Alperin ${ }^{1}$ and Natalia G. Berloff ${ }^{2,1, *}$ \\ ${ }^{1}$ Department of Applied Mathematics and Theoretical Physics, University of Cambridge, Cambridge CB3 OWA, United Kingdom \\ ${ }^{2}$ Skolkovo Institute of Science and Technology, Novaya Street, 100, Skolkovo 143025, Russian Federation
}

(Received 22 May 2019; revised 1 September 2020; accepted 11 September 2020; published 29 September 2020)

\begin{abstract}
We show that exciton-polariton condensates may exhibit a fundamental, self-localized nonlinear excitation in quantum hydrodynamical systems, which takes the form of a dark ring-shaped breather. We predict that these structures form spontaneously and remain stable under a combination of uniform resonant and nonresonant forcing. We study single-ring dynamics, ring interactions, and ring turbulence, and explain how direct experimental observations might be made. We discuss the statistics of ring formation and propose an experimental scheme by which these structures may be exploited to study the smooth crossover between equilibrium and nonequilibrium critical phase transitions. The observation of a breathing-ring soliton would represent a fundamental breathing soliton within the broad field of quantum hydrodynamics.
\end{abstract}

DOI: 10.1103/PhysRevA.102.031304

Spontaneous pattern formation in disordered systems has captivated scientists for generations. Of particular interest are self-localized patterns, which are known as solitons in integrable systems and as solitary waves in nonintegrable systems [1,2]. These can be understood as the fundamental excitations of nonlinear wave systems, and they are typically found in familiar forms across disparate settings. The first to be discovered were of the bright type, which are stabilized by the counterbalancing of dispersion with nonlinear effects. Other solitons are of the dark type and include vortex- and domain-wall-type phase dislocations [3-10]. Nonlinear optical resonators were one of the earliest physical systems in which nonlinear dynamical pattern formation was studied in depth [7]. The results of that field translated to many other systems, such as Bose-Einstein condensates (BECs) of ultracold atoms [8,11] and of magnon [12] systems. One fundamental soliton that can be formed in the nonlinear optical resonator is the so-called ring dark or phase soliton, formed by domain walls that close on themselves to form loops [10]. In equilibrium BECs (such as ultracold atomic BECs), it has been shown that ring dark solitons are not stable, decaying either acoustically or into so-called vortex necklaces $[13,14]$. These structures have been observed experimentally, though fleetingly, by trapping them along their transverse axes [15]. While with this approach the ring structures are localized in that they repeatedly break down and reform within the trap, they are not self-localized and cannot exist in free space.

In this Rapid Communication we show that excitonpolariton condensates-hybrid light-matter quantum fluids with strongly nonlinear properties and inherent nonequilibration-may support a different type of topological defect: breathing-ring solitons. These breathing rings are distinct from other solitary structures found in

\footnotetext{
*Author to whom correspondence should be addressed: N.G.Berloff@damtp.cam.ac.uk
}

exciton-polariton condensates as well as from those found in other quantum hydrodynamical systems. Geometrically these excitations are similar to the ring-shaped phase solitons found in nonlinear optical systems [16], but are distinct in a critical way: the solitons we present here do not appear to settle into a stationary state, instead continuing to oscillate indefinitely in radius and depth [17]. We study the mechanisms of formation and stabilization of these structures, and report on their dynamics and interactions, including states of ring solitonic turbulence. We also study the statistics of spontaneous ring formation, showing that breathing-ring solitons can be used to study critical phenomena in systems with tunable nonequilibration.

Condensates of exciton-polariton quasiparticles (polariton condensates) have recently been realized in semiconductor microcavities [18]. As inherently nonequilibrated condensates of hybrid light-matter quasiparticles, polariton condensates may be thought of neither as equilibrium condensates nor as lasers, but rather as something in between [19]. Polaritonic systems have several advantages with respect to other confined optical systems. One is their extraordinary nonlinear properties, which arise from their excitonic component. Their dispersion curves (the so-called lower and upper polariton branches appearing from the hybridization of photons and excitons) allow for the individual control of the photonic and excitonic components via detuning, and their properties and dynamics can be easily accessed by angular-resolved imaging or electroluminescence spectroscopy. For low enough densities, polaritons may be considered as bosonic quasiparticles, and so can form a coherent state (BEC). Polariton condensates are nonequilibrium systems set by balance between pumping and losses due to the short lifetime of polaritons. They can continuously cross from weak coupling at higher temperatures and pumping strengths to strong coupling at lower temperatures and lower pumping intensities. In addition, the lifetime of polaritons in the microcavity systems can be increased by improving the quality and number of dielectric Bragg mirrors. 
Therefore, depending on the polariton lifetime and pumping intensities, polariton condensates continuously cross between strongly nonequilibrium systems-lasers (short lifetimes and large pumping rates) and equilibrium BECs (large lifetimes and small pumping).

Polariton condensates can be excited by two different types of pumping. In the resonant pumping scheme, the energy and angle of incidence of the excitation laser are set to be quasiresonant with a mode of the lower polariton branch. In the nonresonant pumping scheme, the excitation laser has an energy much higher than that of the lower polariton branch. In this case, polaritons can spontaneously form macroscopic coherent states-polariton lasers or polariton condensates-by the accumulation of particles in the same quantum state [18]. Recent experiments have started to combine resonant and nonresonant pumping [20]. In these experiments, chemical etching of a GaAs substrate has allowed resonant excitation from the back side of the cavity, preventing backscatter from the nonresonant pumping, while allowing synchronization. This technique makes it possible to independently vary the pumping intensity distributions of resonant and nonresonant excitations.

The mean-field behavior of polariton condensates is governed by the generalized complex Ginzburg-Landau equation (cGLE), with the condensate wave function $\psi(\mathbf{r}, t)$ coupled to the hot exciton reservoir density $N_{R}[19,21-24]$, so that

$$
\begin{aligned}
i \partial_{t} \psi= & -\left(1-i \eta N_{R}\right) \nabla^{2} \psi+|\psi|^{2} \psi+g N_{R} \psi \\
& +i\left(N_{R}-\gamma\right) \psi+V_{\mathrm{ext}} \psi+i \bar{P} \psi^{*(n-1)}, \\
& \partial_{t} N_{R}=P-\left(b_{0}+b_{1}|\psi|^{2}\right) N_{R},
\end{aligned}
$$

where we set $\hbar=1$ and $m=1 / 2$. In these coupled equations, $g$ is proportional to the polariton-exciton interaction strength, $\eta$ represents the energy relaxation $[25,26], \gamma$ and $b_{0}$ are proportional to the inverse lifetime of the polariton and hot excitons, respectively, and $b_{1}$ is proportional to the ratio of the interaction strength between the condensate and the hot excitons to that between condensate particles. The incoherent and resonant (at $n: 1$ resonance with the condensate frequency) pump sources are described by the pumping intensities $P(\mathbf{r}, t)$ and $\bar{P}(\mathbf{r}, t)$, respectively [27]. The external resonant forcing described by $\bar{P}$ is at the frequency $\omega_{c}=n \omega_{0}$ where the natural (base) frequency of the condensate is $\omega_{0}[7,28]$. In what follows we will be interested in the second-order resonance $n=2$. In experiment, $2: 1$ resonance can be achieved with the same apparatus used for combined nonresonant and $n=1$ resonant pumping (for example, Ref. [20]), but tuning it to twice the frequency of the condensate, which is within the laser capabilities [29].

The uniform stationary solutions of Eqs. (1) and (2) with $n=2$ and without noise satisfy $0=R^{2}+g \tilde{P} /\left(1+\xi R^{2}\right)+$ $\bar{P} \sin 2 S$ and $\gamma=\tilde{P} /\left(1+\xi R^{2}\right)+\bar{P} \cos 2 S$, where we used the Madelung transformation $\psi=R \exp [i S]$ and denoted $\tilde{P}=$ $P / b_{0}, \xi=b_{1} / b_{0}$. Eliminating $S$ gives $\bar{P}^{2}=\left[R^{2}+g \tilde{P}(1+\right.$ $\left.\left.\xi R^{2}\right)^{-1}\right]^{2}+\left[\gamma-\tilde{P}\left(1+\xi R^{2}\right)^{-1}\right]^{2}$. For given system parameters this equation can be solved to find $\rho=R^{2}$ with two expressions for $S$ that differ by $\pi$.

The regime of $R \in \mathbb{R}$ then corresponds to the regime of phase-bistable, frequency-locked solutions; it is in this regime that Ising domain walls may exist. These are the one-dimensional zeros which separate regions of differing phase. Ising walls are required to either end at the boundary of the condensate or to form closed loops that grow or shrink until they reach a characteristic radius. In the case of the nonlinear optical resonator (as in Ref. [10]) such rings are stable, minimizing the local potential energy, and thus remain stationary. However, after reaching the critical radius the rings in polariton condensates behave differently. We determined that the ring solitons self-annihilate (i) in the fast reservoir regime $b_{0} \gg \gamma$, (ii) in a small reservoir detuning regime $g \ll 1$, and (iii) in condensates made of long-lived polaritons. All these regimes are physically relevant to some experiments [30-32]. However, a slow reservoir evolution $\left(b_{0} \lesssim \gamma\right)$, for short-lived polaritons and a sufficiently large reservoir detuning (all of which correspond to values of typical GaAs microcavity experiments $[18,33,34]$ ), prevent the ring soliton from disappearing and lead to the appearance of a ring breather: the dissipative decrease in the radius of the ring soliton is accompanied by the increase in the reservoir profile density in the ring core, which imposes a repulsive force in the outward direction to make the ring expand. This process repeats itself as shown in Supplemental Material Video 1 [35]. This nonlinear excitation is self-localized by an explicitly dynamical interaction.

While the existence of a closed-form description of this dynamical structure is highly unlikely, some insight into its structure can be gained by treating the breathing behavior as a perturbation of a stationary solution. Using the Taylor expansion in the steady state expression for the reservoir density, $N_{R}=\tilde{P}\left(1+\xi|\psi|^{2}\right)^{-1} \approx \tilde{P}-\tilde{P} \xi|\psi|^{2}$. The dynamics of the condensate results from Eqs. (1) and (2) and reads

$\partial_{t} \psi=(i+\eta \tilde{P}) \nabla^{2} \psi-\kappa|\psi|^{2} \psi+\bar{P} \psi^{*}+[(g \tilde{P}) i+\tilde{P}-\gamma] \psi$,

where we denoted $\kappa=\tilde{P} \xi+(1-g \tilde{P} \xi) i$.

Close to the condensation threshold and for sufficiently strong external resonant forcing, we can assume that $\eta \tilde{P} \ll 1$. Neglecting the corresponding terms, rescaling $\psi \rightarrow \Psi \sqrt{\bar{P} / \tilde{P} \xi}$, $t \rightarrow t / \bar{P}, \mathbf{x} \rightarrow \mathbf{x} / \sqrt{\bar{P}}$, and denoting $\chi=(1-g \tilde{P} \xi) / \tilde{P} \xi, \alpha_{1}=$ $(\tilde{P}-\gamma) / \bar{P}$, and $\alpha_{2}=g \tilde{P} / \bar{P}$, we rewrite Eq. (3) as

$$
\partial_{t} \Psi_{i}=i \nabla^{2} \Psi-(1+i \chi)|\Psi|^{2} \Psi+\Psi^{*}+\left(\alpha_{1}-i \alpha_{2}\right) \Psi .
$$

The uniform density is given by $|\Psi|^{2}=1+\alpha_{1}$. We rewrite the condensate wave function as the sum of real and imaginary components $\Psi=U+i V$, so that

$$
\begin{aligned}
\partial_{t} U= & -\nabla^{2} V-\left(U^{2}+V^{2}\right) U+\chi\left(U^{2}+V^{2}\right) V \\
& +\left(1+\alpha_{1}\right) U+\alpha_{2} V, \\
\partial_{t} V= & \nabla^{2} U-\left(U^{2}+V^{2}\right) V-\chi\left(U^{2}+V^{2}\right) U \\
& -\left(1-\alpha_{1}\right) V-\alpha_{2} U .
\end{aligned}
$$

In the ring soliton $V \ll U$ and so $|\Psi|^{2} \approx U^{2} \approx 1+\alpha_{1}$ except for the small healing region that defines the radius of the ring, and $\partial_{t} V \approx 0$. Under these assumptions we solve Eq. (6) for $V \approx\left[\nabla^{2} U-\left\{\chi\left(1+\alpha_{1}\right)+\alpha_{2}\right\} U\right] / 2$ and substitute into 
Eq. (5) to get a real Swift-Hohenberg equation (RSHE)

$$
\partial_{t} U=-\frac{1}{2}\left[\nabla^{2}+\Delta\right]^{2} U-U^{3}+\left(1+\alpha_{1}\right) U,
$$

where we write $\Delta=-\left[\chi\left(1+\alpha_{1}\right)+\alpha_{2}\right]$.

RSHE is a variational equation, and can be written in the gradient form $\partial_{t} U=-\partial \mathcal{F} / \partial U$ [36], where the potential $\mathcal{F}$ takes the form $\mathcal{F}=\int_{0}^{+\infty}\left\{\frac{1}{4}\left[\left(\nabla^{2}+\Delta\right) U\right]^{2}-\frac{1}{4}\left(U^{4}-U_{0}^{4}\right)-\right.$ $\left.\frac{1}{2}\left(1+a_{1}\right)\left(U^{2}-U_{0}^{2}\right)-\frac{1}{4} \Delta^{2} U_{0}^{2}\right\} d r$, denoting the background contribution as $U_{0}=\sqrt{1+\alpha_{1}-1 / 2 \Delta^{2}}$.

In one dimension the Ising wall takes the approximate form $U(r)=U_{0} \tanh (r / w)$, where the width parameter $w$ determines the healing length. Inserting this into $\mathcal{F}$ and evaluating the integral yields an analytical form for the potential energy. The Ising wall width $w$ which minimizes this potential can be written as

$$
w^{2}=\frac{\sqrt{5} \Delta^{2}+12 U_{0}^{2}}{\sqrt{5} U_{0}^{2}}-\frac{\Delta}{U_{0}^{2}} .
$$

The circularly symmetric Ising wall can be approximated with the ansatz $U(r)=U_{0}\left(r+r_{0}\right)\left(r-r_{0}\right) /$ $\sqrt{\left[\left(r+r_{0}\right)^{2}+w^{2}\right]\left[\left(r-r_{0}\right)^{2}+w^{2}\right]}$. Substituting $w$ and inserting the two-dimensional (2D) ansatz (again taking care to remove the contribution of the homogenous background), $\mathcal{F}$ is again exactly integrable, yielding an analytical formula for the potential energy of the ring soliton as a function of its radius and the system parameters. This potential takes the exact form

$$
\begin{aligned}
\mathcal{F}= & \frac{U_{0}^{2}}{1024 r_{0}^{5} w^{3}\left(r_{0}^{2}+w^{2}\right)^{4}}\left\{r _ { 0 } w \left(-27 w^{12}\right.\right. \\
& -4 r_{0}^{12}\left(-45+96 \Delta w^{2}-64 V^{2} w^{4}\right) \\
& -r_{0}^{2} w^{10}\left(229+32 \Delta w^{2}+16 V^{2} w^{4}\right) \\
& +2 r_{0}^{4} w^{8}\left(-241-192 \Delta w^{2}+56 V^{2} w^{4}\right) \\
& +2 r_{0}^{6} w^{6}\left(127-608 \Delta w^{2}+328 V^{2} w^{4}\right) \\
& +r_{0}^{10} w^{2}\left(807-1312 \Delta w^{2}+896 V^{2} w^{4}\right) \\
& \left.+r_{0}^{8} w^{4}\left(1545-1792 \Delta w^{2}+1168 V^{2} w^{4}\right)\right) \\
& +\left(r_{0}^{2}+w^{2}\right)^{4}\left[27 w^{6}-4 r_{0}^{6}\left(-45+96 \Delta w^{2}-64 V^{2} w^{4}\right)\right. \\
& +2 r_{0}^{2} w^{4}\left(65+16 \Delta w^{2}+8 V^{2} w^{4}\right) \\
& \left.\left.+r_{0}^{4} w^{2}\left(147+96 \Delta w^{2}+128 V^{2} w^{4}\right)\right] \arctan \left(r_{0} / w\right)\right\} .
\end{aligned}
$$

For typical system parameters, Fig. 1 shows the potential energy $\mathcal{F}$ of the ring as a function of its radius, for several values of the pump power. This shows that under the approximation of the breathing-ring soliton's radial oscillations as small perturbations of a stationary solution, a significant amount of insight can be gleaned about the relationship between the pump strength and the behavior of the ring: too small, and the ring expands to infinity, but too large, and the ring contracts to a point. As the inset shows, for a range of values in between, there exist finite ring radii which minimize the potential. Over this range, the minimizing radius decreases with increasing pump power. The steepness of the curve also decreases towards the large $r_{0}$ side as the pump is decreased, suggesting that as the pump strength is lowered, the range of

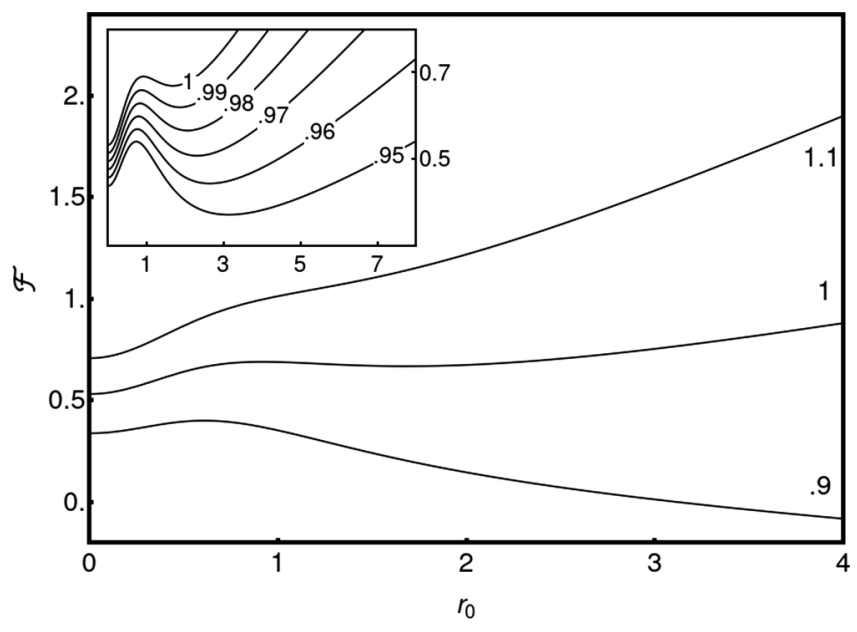

FIG. 1. The potential energy $\mathcal{F}$ as a function of the ring radius $r_{0}$, for several pump powers $P=\bar{P}$, for $g=1$. The inset shows the same, for a smaller range of pump strength, for which there exist energy minima for finite ring radii.

$r_{0}$ over the ring oscillation should increase. While the relevant range of pump strengths differs between the analytical predictions and the full numerical experiments, the behavioral predictions of the analytical model are entirely consistent with the numerical experiments.

The destabilizing mechanisms (i)-(iii) have similar effects on the existence and dynamics of the ring solitons, so we concentrate on the effect of varying the polariton-exciton interaction strength (parametrized by $g$ ). The detuning between the cavity photon energy and the exciton resonance determines the relative photonic and excitonic character of the polariton and, therefore, its effective mass and the strength of the polariton-exciton interactions [23]. The detuning $g$ can be further changed by the pumping geometry by considering trapped condensates separated from the pumps [37]. Finally, implanting protons into the quantum wells or into the top of distributed Bragg reflectors allows for an independent spatial control of both the exciton and the cavity photon energies, and, therefore, affects $g$ [38] as well. By these mechanisms, the experimental ranges of our dimensionless parameter $g$ can vary between 0.1 and 1.5 . We observe spontaneous ring formation in the entire physical range of that parameter. It is found that the pumping amplitudes for which ring formation is supported depends on the detuning, extending for a range of nearly $P_{\text {th }}$ for the case $g=1$, and extending for a range of more than $2 P_{\text {th }}$ for $g=0.1$. We note that the experimental range of values of $\eta$ are unknown. However, we have observed ring formation for the range $0.001 \leqslant \eta \leqslant 1$.

In systems with low $g$, rings form ad infinitum creating a sustained state of ring turbulence. A time snapshot of condensate density in this regime is shown in Fig. 2(a). In the high $g$ case, rings are formed only during the condensation process. They tend to interact attractively, and upon contact a pair of rings appear to either merge into one or annihilate each other. Eventually the decay of rings ends and a quasistationary state is reached with rings being pinned by the system disorder represented in our simulations by setting $V_{\text {ext }}$ to white noise with amplitudes ranging between $\pm 0.005 \rho_{0}$, where $\rho_{0}$ 

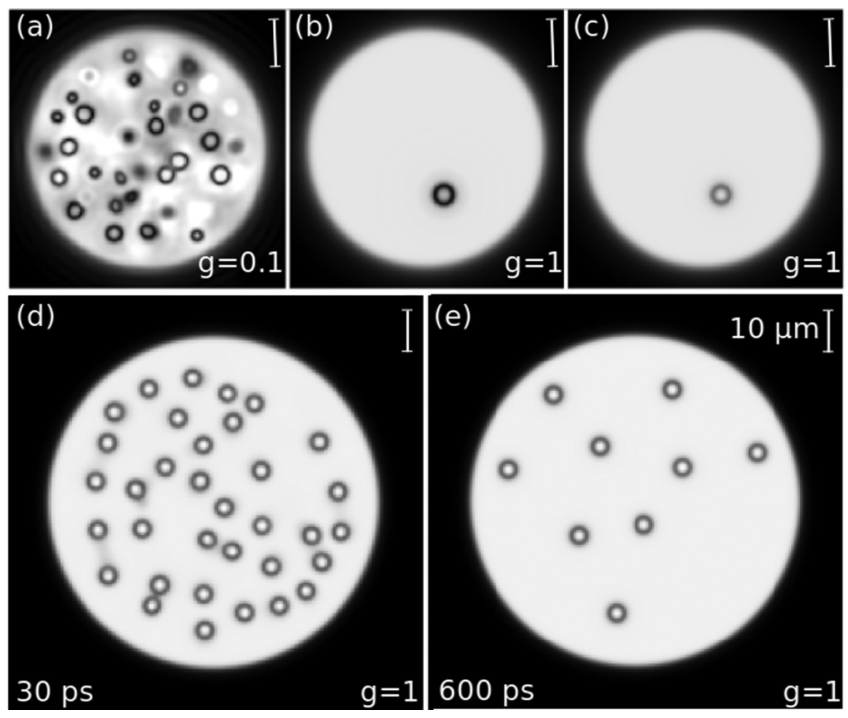

FIG. 2. Spontaneously formed breathing rings in excitonpolariton condensates for $P=\bar{P}=5$, where $P$ is the amplitude of the nonresonant pump, and $\bar{P}$ is that of a second-order resonant pump. Density contour plots of the condensate shown illustrate (a) a time snapshot of ring turbulence, (b) a quasistationary state with a single ring, (c) time averaging of (b) over many ring oscillations, and (d), (e) different stages of the condensate evolution averaged over the timescale of the ring oscillation.

is uniform density profile. However, we note that we see the same type of pinning behavior for $V_{\mathrm{ext}}=0$, as even when the only disorder comes from the discretization of the fluid (the high-energy limit of any numerical simulation, and of any physical many body system), structures that are far enough apart interact so negligibly that they remain stationary, pinned by slightly less negligible disorder (at least up to timescales relevant to experimental observation). This is demonstrated in Supplemental Material Video 2 [35], with time snapshots of the condensate density shown in Figs. 2(d) and 2(e). Figure 2 shows a time snapshot of a spontaneously formed breathing-ring soliton after the system has reached its final, quasistationary state [Fig. 2(b)], as well as a time-integrated image of that state [Fig. 2(c)]. Further, we note that we find the rings to be stable against finite noise. Thus we predict that long-lived breathing-ring solitons are directly observable, and that their ring-shaped character, radii, locations, and numbers are directly measurable as well.

The mechanism by which breathing-ring solitons have been shown to form for high $g$ resembles the Kibble-Zurek (KZ) mechanism of defect formation in equilibrium systems $[39,40]$. The KZ mechanism was first understood in the context of the phase transitions in the early Universe [41-43], and later in liquid ${ }^{4} \mathrm{He}$ and ${ }^{3} \mathrm{He}$, liquid crystals, superconductors [44-47], and equilibrium Bose-Einstein condensates [48,49]. The similarities and differences between the KZ transition and pattern formation in nonequilibrium systems are the subject of intense exploration, with an emphasis on the common mechanism of the defect formation: locally uniform symmetry breaking in separate parts of the system which cannot communicate in a finite time, and which thus form to be globally nonuniform to a degree set by the speed of the phase transition (the quench rate). The main difference between the

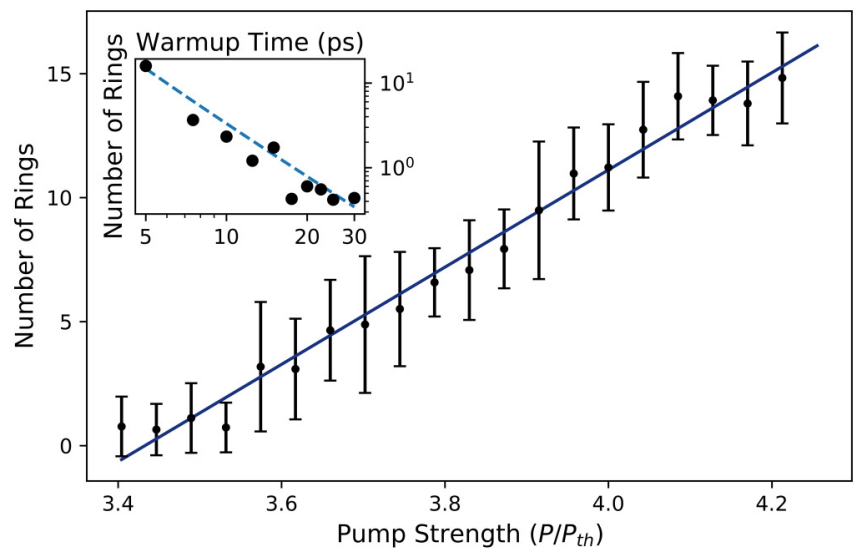

FIG. 3. Number of rings in the quasistationary state as a function of pump strength $(P=\bar{P}=$ const $)$, in units of the threshold pump strength $P_{\mathrm{th}}$. Results are averaged over ten random iterations of initial noise and potential disorder. A linear fit is shown in blue. The inset shows a log-log plot of the number of rings in the quasistationary state as a function of warmup time, defined as the time over which the pumps are increased to a fixed amplitude $\left(P=\bar{P}=4.2 P_{\mathrm{th}}\right)$. A dashed blue line shows the power law $t^{-2}$.

$\mathrm{KZ}$ transition and pattern forming in nonequilibrium systems is that in the former, it is assumed that the system is driven out of equilibrium only in the vicinity of the phase transition [43]. In spite of extensive research on both the $\mathrm{KZ}$ transition and on pattern formation in a wide variety of nonequilibrium systems, questions remain regarding the nature of the crossover between the two mechanisms, and regarding the types of the defects that they can result in. Numerical experiments regarding the rate of polarization defect formation between quasi-1D spinor polariton condensates formed in chains of microcavities have been performed [50], but to our knowledge no proposal of this kind of study has been made in regard to uniform polariton condensates formed on ordinary GaAs samples, or in regard to phase defects.

We investigate this relationship by counting the number of quasistationary rings formed spontaneously from random initial noise in the presence of a small sample disorder, modeled by setting $V_{\text {ext }}$ to a randomly distributed set of needlelike potentials, with a Gaussian profile and width much smaller than the healing length of the condensate. This disorder does not hamper the formation of rings, but rather acts as sandpaper, resisting their movement across its surface. Figure 3 shows the resulting linear, positive correlation between pump power and ring soliton density. To elucidate the effect of the quenching time on the defect formation, we repeated our simulations linearly increasing the pumps from zero to $P=\bar{P}$ over different timescales. The results, shown in the inset of Fig. 3, reveals a $t^{-2}$ power law. We note that recent theoretical work on nonequilibrium holographic superfluids has shown qualitatively similar results: a linear dependence of excitation strength (temperature in that context) on defect (vortex) density, and a power-law dependence of quench time on defect density [51].

In conclusion, we have theoretically predicted the spontaneous formation of stable breathing-ring solitons in excitonpolariton condensates. The proposed experimental realization 
for such topological defects is well within the current experimental conditions and properties of existing microcavities. These structures represent a fundamental breather and a stable ring soliton in a quantum hydrodynamical system, and are made possible by the polariton condensates' unique combination of inherent nonequilibration with the existence of a hot exciton reservoir which scatters particles into the condensate while repulsively interacting with condensed particles. We have shown how combining resonant and nonresonant forcing can be used to suppress the snake instability, and have discussed how the robust stability of breathing-ring solitons can be exploited to study nonequilibrium defect formation statistics, and thus to probe the fundamentals of nonequilibrium phase transitions. Further, we have proposed an experimental scheme by which these statistics could be probed over the continuous crossover between equilibrium and nonequilibrium phase transitions. This work highlights the exceptional promise of exciton-polariton condensates in the highly interdisciplinary field of nonlinear pattern formation.

N.G.B. acknowledges the support from Huawei. Both authors are grateful to Kirill Kalinin for discussions.
[1] G. B. Whitham, Linear and Nonlinear Waves (Wiley, Hoboken, NJ, 2011), Vol. 42.

[2] M. J. Ablowitz and H. Segur, Solitons and the Inverse Scattering Transform (SIAM, Philadelphia, 1981), Vol. 4.

[3] M. Berry and M. Dennis, Proc. R. Soc. London, Ser. A 456, 2059 (2000).

[4] S. N. Alperin, A. L. Grotelueschen, and M. E. Siemens, Phys. Rev. Lett. 122, 044301 (2019).

[5] A. Vilenkin, Phys. Rep. 121, 263 (1985).

[6] L. M. Pismen and L. M. Pismen, Vortices in Nonlinear Fields: From Liquid Crystals to Superfluids, From Non-Equilibrium Patterns to Cosmic Strings, International Series of Monographs on Physics Vol. 100 (Oxford University Press, Oxford, UK, 1999).

[7] L. M. Pismen, Patterns and Interfaces in Dissipative Dynamics (Springer, Berlin, 2006).

[8] S. Burger, K. Bongs, S. Dettmer, W. Ertmer, K. Sengstock, A. Sanpera, G. V. Shlyapnikov, and M. Lewenstein, Phys. Rev. Lett. 83, 5198 (1999).

[9] G. A. Swartzlander, Jr. and C. T. Law, Phys. Rev. Lett. 69, 2503 (1992).

[10] K. Staliunas and V. J. Sanchez-Morcillo, Transverse Patterns in Nonlinear Optical Resonators (Springer, Berlin, 2003), Vol. 183.

[11] P. G. Kevrekidis, D. J. Frantzeskakis, and R. CarreteroGonzález, Emergent Nonlinear Phenomena in Bose-Einstein Condensates: Theory and Experiment (Springer, Berlin, 2007), Vol. 45.

[12] P. Nowik-Boltyk, O. Dzyapko, V. Demidov, N. Berloff, and S. Demokritov, Sci. Rep. 2, 482 (2012).

[13] L. D. Carr and C. W. Clark, Phys. Rev. A 74, 043613 (2006).

[14] G. Theocharis, D. J. Frantzeskakis, P. G. Kevrekidis, B. A. Malomed, and Y. S. Kivshar, Phys. Rev. Lett. 90, 120403 (2003).

[15] I. Shomroni, E. Lahoud, S. Levy, and J. Steinhauer, Nat. Phys. 5, 193 (2009).

[16] V. B. Taranenko, K. Staliunas, and C. O. Weiss, Phys. Rev. Lett. 81, 2236 (1998).

[17] We do note that it has been predicted, in the exceptional circumstances of high nonlinearity and nonlocality, breathing optical ring-shaped solitons may exist [52]. However, these solitons are of the beam type as opposed to the systems of interest here (self-localized structures within an extended system). Thus these are of a distinct class of structure.
[18] J. Kasprzak, M. Richard, S. Kundermann, A. Baas, P. Jeambrun, J. Keeling, F. Marchetti, M. Szymańska, R. André, J. Staehli et al., Nature (London) 443, 409 (2006).

[19] J. Keeling and N. G. Berloff, Phys. Rev. Lett. 100, 250401 (2008).

[20] H. Ohadi, Y. del Valle-Inclan Redondo, A. Dreismann, Y. G. Rubo, F. Pinsker, S. I. Tsintzos, Z. Hatzopoulos, P. G. Savvidis, and J. J. Baumberg, Phys. Rev. Lett. 116, 106403 (2016).

[21] K. P. Kalinin and N. G. Berloff, Phys. Rev. Lett. 121, 235302 (2018).

[22] M. Wouters and I. Carusotto, Phys. Rev. Lett. 99, 140402 (2007).

[23] I. Carusotto and C. Ciuti, Rev. Mod. Phys. 85, 299 (2013).

[24] J. Keeling and N. G. Berloff, Contemp. Phys. 52, 131 (2011).

[25] M. Wouters, New J. Phys. 14, 075020 (2012).

[26] N. Berloff and J. Keeling, in Physics of Quantum Fluids (Springer, Berlin, 2013), pp. 19-38.

[27] The fixed parameter values in our simulations are $b_{0}=1$, $b_{1}=1, \gamma=0.3$, and $\eta=0.3$.

[28] P. Coullet and K. Emilsson, Physica D 61, 119 (1992).

[29] P. Savvidis and H. Ohadi (private communication).

[30] B. Nelsen, G. Liu, M. Steger, D. W. Snoke, R. Balili, K. West, and L. Pfeiffer, Phys. Rev. X 3, 041015 (2013).

[31] K. P. Kalinin and N. G. Berloff, Phys. Rev. B 100, 245306 (2019).

[32] N. G. Berloff, M. Silva, K. Kalinin, A. Askitopoulos, J. D. Töpfer, P. Cilibrizzi, W. Langbein, and P. G. Lagoudakis, Nat. Mater. 16, 1120 (2017).

[33] H. Deng, G. S. Solomon, R. Hey, K. H. Ploog, and Y. Yamamoto, Phys. Rev. Lett. 99, 126403 (2007).

[34] F. Manni, K. G. Lagoudakis, R. André, M. Wouters, and B. Deveaud, Phys. Rev. Lett. 109, 150409 (2012).

[35] See Supplemental Material at http://link.aps.org/supplemental/ 10.1103/PhysRevA.102.031304 for Videos.

[36] M. C. Cross and P. C. Hohenberg, Rev. Mod. Phys. 65, 851 (1993).

[37] P. Cristofolini, A. Dreismann, G. Christmann, G. Franchetti, N. G. Berloff, P. Tsotsis, Z. Hatzopoulos, P. G. Savvidis, and J. J. Baumberg, Phys. Rev. Lett. 110, 186403 (2013).

[38] C. Schneider, K. Winkler, M. Fraser, M. Kamp, Y. Yamamoto, E. Ostrovskaya, and S. Höfling, Rep. Prog. Phys. 80, 016503 (2016).

[39] B. Damski and W. H. Zurek, Phys. Rev. Lett. 104, 160404 (2010). 
[40] W. H. Zurek, Phys. Rep. 276, 177 (1996).

[41] T. W. Kibble, J. Phys. A: Math. Gen. 9, 1387 (1976).

[42] T. W. Kibble, Phys. Rep. 67, 183 (1980).

[43] W. H. Zurek, Nature (London) 317, 505 (1985).

[44] C. Bäuerle, Y. M. Bunkov, S. Fisher, H. Godfrin, and G. Pickett, Nature (London) 382, 332 (1996).

[45] V. Ruutu, V. Eltsov, A. Gill, T. Kibble, M. Krusius, Y. G. Makhlin, B. Placais, G. Volovik, and W. Xu, Nature (London) 382, 334 (1996).

[46] M. J. Bowick, L. Chandar, E. A. Schiff, and A. M. Srivastava, Science 263, 943 (1994).
[47] A. Maniv, E. Polturak, and G. Koren, Phys. Rev. Lett. 91, 197001 (2003).

[48] N. G. Berloff and B. V. Svistunov, Phys. Rev. A 66, 013603 (2002).

[49] C. N. Weiler, T. W. Neely, D. R. Scherer, A. S. Bradley, M. J. Davis, and B. P. Anderson, Nature (London) 455, 948 (2008).

[50] D. D. Solnyshkov, A. V. Nalitov, and G. Malpuech, Phys. Rev. Lett. 116, 046402 (2016).

[51] P. M. Chesler, A. M. García-García, and H. Liu, Phys. Rev. X 5, 021015 (2015).

[52] D. Lu and W. Hu, Phys. Rev. A 79, 043833 (2009). 